\title{
Differences between Tempos of Step Error and Postural Sway in the Stipulated Tempo Step Test for Children and Their Relationships
}

\author{
Hiroki Aoki ${ }^{1,}{ }^{*}$, Shin-ichi Demura ${ }^{2}$, Kenji Takahashi ${ }^{3} \&$ Hiroshi Hirai $^{4}$ \\ ${ }^{1}$ National Institute of Technology, Fukui College, Fukui, Japan \\ ${ }^{2}$ Kanazawa University, Ishikawa, Japan \\ ${ }^{3}$ Nagasaki International University, Nagasaki, Japan \\ ${ }^{4}$ Osaka Prefecture University, Osaka, Japan \\ *Correspondence: National Institute of Technology, Fukui College, Geshi, Sabae, Fukui 916-8507, Japan. Tel: \\ 81-778-62-1111. E-mail: aoki@fukui-nct.ac.jp
}

Received: May 31, 2021

doi:10.5430/wje.v11n6p31
Accepted: November 25, $2021 \quad$ Online Published: December 6, 2021

URL: https://doi.org/10.5430/wje.v11n6p31

\begin{abstract}
Recently, to evaluate dynamic balance ability, a stipulated tempo step test has been developed, and a step error between tempo and contact time of feet has been used as an evaluation variable. The step error, postural sway, and their relationships may differ between the slow tempo $(40 \mathrm{bpm})$ and fast tempo $(120 \mathrm{bpm})$. This study aimed to examine the aforementioned problem with 62 participant children ( 30 boys and 32 girls). The step error and postural sway variables (X-axis path length, Y-axis path length, total path length, peripheral area, and rectangular area) during stepping while matching both tempos were measured. Means of one minute and three intervals $(0-20 \mathrm{sec}, 20-40 \mathrm{sec}$, and $40-60 \mathrm{sec}$ ) for each variable were calculated in both tempos. The results of the paired t-test showed that means of all variables were larger in the $40 \mathrm{bpm}$ tempo than in the $120 \mathrm{bpm}$ tempo. In the multiple comparison tests after the results of the two-way repeated measures ANOVA, the means of three intervals in all variables were larger in the $40 \mathrm{bpm}$ tempo than in the $120 \mathrm{bpm}$ tempo; the means of the sway variables, excluding that of the $\mathrm{X}$-axis path length, in the $40 \mathrm{bpm}$ tempo were larger in the 0-20 sec interval than in the 20-40 sec interval or the 40-60 sec interval. Correlations between step errors and those between the step error and sway variables of both tempos were insignificant or under moderation. The correlations between the step error and sway variables in both tempos were insignificant or significant but low, and those among sway variables were high, except between the $\mathrm{X}$ - and Y-axis path lengths. The relationship between both axis path lengths differed according to the tempo.

In conclusion, in the case of the stipulated tempo step test targeting children, the slow tempo has a greater step error and postural sway than the fast tempo, and the sway in the early step stage is greater in the slow tempo. The relationships between step errors and between the step error and sway variables of both tempos are low; hence, the ability related to the test may differ in both tempos. The relationships among sway variables in both tempos are high, except between the $\mathrm{X}$ - and Y-axis path lengths.
\end{abstract}

Keywords: children, stipulated tempo, step

\section{Introduction}

Dynamic balance ability refers to maintaining the stability of the posture during body movement and has been evaluated in targeting subjects of a wide age range from infants to the elderly (Shin \& Demura, 2009; Aoki et al., 2012). A stipulated tempo step test recently developed by Shin and Demura (2009) has been used a time difference between tempo and contact time of feet (step error) as an evaluation variable based on the assumption that people can step while matching the tempo suitably, thereby having superior dynamic balance ability (Shin \& Demura, 2009; Aoki et al., 2012). Shin and Demura (2007) reported that the mean of step error in the stipulated tempo step test is larger in the order of 40,60, and $120 \mathrm{bpm}$ tempos in elderly subjects, but an insignificant difference was observed among tempos in young adults. In addition, the means of 40 and $60 \mathrm{bpm}$ tempos were larger in the elderly than in the young. Toyama et al. (1990) reported that the $120 \mathrm{bpm}$ is the tempo in which people can step the steadiest because 
the walking pace is approximated. The $40 \mathrm{bpm}$ is considerably a slower tempo than the $120 \mathrm{bpm}$. The slower the tempo is, the longer the time supported by only one leg during stepping. Elderlies with inferior leg strength have a difficult time keeping a stable posture. Hence, they cannot step suitably while matching the tempo. Likewise, children with underdeveloped leg strength have a hard time stepping while matching the slow tempo; therefore, they may have a large step error.

Previous studies on postural sway have used sway components regarding distance, speed, and area as postural sway variables (Demura et al., 2008; Maribo et al., 2011; Suzuki et al., 2015). Aoki et al. (2012) recorded that postural sway (e.g., peripheral area and rectangular area) during stepping with the $100 \mathrm{bpm}$ tempo is larger in elderly subjects than in young adults, suggesting that the elderly with inferior leg strength have a larger postural sway than young adults. An unsteady posture during stepping also reflects a postural sway, that is, it is assumed that because the slower the tempo due to the longer the time supported the body by only one leg becomes unstable and postural sway does larger. However, this problem has not been fully examined. In contrast, the postural sway has basically been measured for one minute (Demura et al., 2008; Hara et al., 2007; Demura et al., 2015). In the case of a similar stepping tempo to walking, subjects can continue stepping steadily from the measurement start, but when enforced to step with an unfamiliar and slow tempo, they may feel difficulty in stepping while matching the tempo during the early stage of measurement; hence, the step error and postural sway may become large. The above statement is considered to be prominent in children and elderly people with inferior leg strength.

Meanwhile, relationships among postural sway variables during static standing (Demura et al., 2001) or their fluctuations during static standing with eyes open or closed (Smith et al., 2012; Apthorp et al., 2014; WaltersStewart et al., 2018) have been examined. Yasuda et al. (2012) examined their relationships during stepping with the $120 \mathrm{bpm}$ tempo and reported that high relationships are found. Uchida and Nagura (2014) examined their relationships when ascending/descending steps and while one-leg standing.

However, the relationships of the speed or accuracy of movement and body sway have been rarely studied. If the one-leg standing posture due to stepping is unsteady, then the step error and postural sway become large. In addition, as previously mentioned, given that the time during one-leg standing differs considerably between the 40 and 120 bpm tempos, relationships among the step error and sway variables also differ in both tempos.

This study aimed to examine differences between the step error and postural sway variables in a stipulated tempo step test for one minute with a slow tempo $(40 \mathrm{bpm})$ and fast tempo $(120 \mathrm{bpm})$ for children, including the relationships among these variables.

\section{Method}

\subsection{Subjects}

The subjects were 62 (boys: 30 and girls: 32) school children. Age and weight showed insignificant differences between gender groups, but height exhibited a significant difference. We explained to the children and their parents the purpose, methods, and risks of the experiment and eventually obtained their consent. The present protocol was approved by the National Institute of Technology, Fukui College.

Table 1. Basic Static of Participants Age Height and Weight and the Results of the t-Test

\begin{tabular}{llllll}
\hline & boy & & girl & \\
\hline Mean & SD & & Mean & SD & t-value \\
age & 7.8 & 1.5 & 8.5 & 1.5 & 1.921 \\
height $(\mathrm{cm})$ & 129.2 & 8.4 & 134.5 & 8.2 & 2.515 \\
weight $(\mathrm{kg})$ & 27.7 & 5.1 & 29.563 & 5.4 & 1.421 \\
\hline
\end{tabular}

$\mathrm{P}<0.05$ 
Table 2. Basic Statistics of the Step Error and Postural Sway Variables and the Results of the t-Test

\begin{tabular}{|c|c|c|c|c|c|c|c|c|c|c|}
\hline & \multicolumn{5}{|c|}{ 40bpm } & \multicolumn{5}{|c|}{$120 \mathrm{bpm}$} \\
\hline & \multicolumn{2}{|c|}{ boys } & \multicolumn{2}{|c|}{ girls } & \multirow[b]{2}{*}{ t-value } & \multicolumn{2}{|c|}{ boys } & \multicolumn{2}{|c|}{ girls } & \multirow[b]{2}{*}{ t-value } \\
\hline & Mean & SD & Mean & SD & & Mean & SD & Mean & SD & \\
\hline step error (sec) & 0.22 & 0.10 & 0.22 & 0.12 & 0.217 & 0.09 & 0.03 & 0.09 & 0.05 & 0.240 \\
\hline $\mathrm{X}$-axis path length $(\mathrm{mm})$ & 146.8 & 16.4 & 148.1 & 19.4 & 0.280 & 117.6 & 12.0 & 121.1 & 11.9 & 1.157 \\
\hline Y-axis path length (mm) & 102.1 & 25.7 & 94.9 & 22.0 & 1.184 & 82.5 & 28.8 & 77.2 & 29.0 & 0.713 \\
\hline total path length (mm) & 198.9 & 28.1 & 194.3 & 26.2 & 0.669 & 157.3 & 22.5 & 156.8 & 20.8 & 0.084 \\
\hline peripheral area $\left(\mathrm{mm}^{2}\right)$ & 762.9 & 157.7 & 726.7 & 182.1 & 0.834 & 320.7 & 82.5 & 303.5 & 71.5 & 0.881 \\
\hline rectangular area $\left(\mathrm{mm}^{2}\right)$ & 1019.8 & 217.4 & 726.7 & 182.1 & 0.974 & 425.8 & 109.8 & 303.5 & 71.5 & 1.138 \\
\hline
\end{tabular}

\subsection{Measurement Instrument and Test}

The dynamic/static balance measuring system (From TAKEI SCIENTIFIC INSTRUMENTS CO., LTD.) was used as the measuring instrument for the stipulated tempo step test. This instrument inputs the information when the subject's foot touched the sheet into the computer as a digital signal. At the same time, it can calculate the time difference (step error) between the tempo and the contact with the feet from their signals. Moreover, the instrument can calculate the center of pressure of the vertical load as the center of foot pressure from the values of the built-in vertical load sensors. The data were recorded in a computer with a sampling frequency at $20 \mathrm{~Hz}$.

The stipulated tempo step test was performed by the following procedure. The tester instructed subjects the following:

(1) Standing on the plate with their hands by their body. (2) Watching the indicator set before their eyes while stepping. (3) Stepping for one minute while matching the beeping sound of the specific tempo generated by the metronome.

In this study, we selected the $120 \mathrm{bpm}$ tempo, which is the most energy efficient during walking (Toyama, 1990), as the fast tempo, and the $40 \mathrm{bpm}$ tempo as the slow tempo, referring to a previous study (Shin \& Demura, 2007). The one-leg standing time while stepping is a $1.5 \mathrm{sec}$ for the $40 \mathrm{bpm}$ tempo and a $0.5 \mathrm{sec}$ for the $120 \mathrm{bpm}$ tempo. The former is three times of the latter. Subjects conducted two trials for each tempo after one practice.

\subsection{Step Error and Postural Sway Variables}

Step errors are time differences between stipulated tempo sound and grounded time of the step foot for one minute. They were calculated 40 for the $40 \mathrm{bpm}$ tempo and 120 for the $120 \mathrm{bpm}$ tempo. In this study, we used the mean of the total time of their absolute values as an evaluation variable. X-axis path length, Y-axis path length, total path length, peripheral area, and rectangular area were selected as postural sway variables, referring to previous studies (Aoki et al., 2012).

To examine the hypothesis that an effect, that is, slow and fast tempos affect the step error and the postural sway, is found from the early stage of the measurement, we calculated the means of three intervals (early [0-20 sec], middle [20-40 sec], and last [40-60 sec]) for each variable. The means of two trials were used as the representative values of each variable. Moreover, the data of male and female subjects were pooled and analyzed together because no significant sex differences were observed in the step error and postural sway variables (Table 2) in addition to age.

\subsection{Research Hypothesis}

In this study, regarding the step error and the postural sway while stepping for one minute using the 40 and $120 \mathrm{bpm}$ tempos, we set the following hypotheses:

Hypothesis 1: The step error and the postural sway while stepping differ between both tempos.

Hypothesis 2: The content of Hypothesis 1 can be found from the early interval $(0-20 \mathrm{sec})$.

Hypothesis 3: In the $40 \mathrm{bpm}$ tempo, the step error and the postural sway are larger in the early interval than in the middle and/or the last intervals.

Hypothesis 4: Relationships between step errors and among postural sway variables while stepping in both tempos differ.

Hypothesis 5: Interrelationships among the step error and postural sway variables while stepping in both tempos 
differ.

\subsection{Analysis Method}

A repeated t-test was conducted to clarify mean differences between both tempos for the step error and postural sway variables. To examine the degree of the mean difference, an effect size (ES) was calculated. A repeated two-way ANOVA was performed to examine mean differences among tempos and intervals.

When showing a significant interaction or a significant main effect, Tukey's Honestly Significant Difference test was used for multiple comparisons. Pearson's correlation was calculated to examine relationships among evaluation variables. The statistical significance was set at the $5 \%$ level.

\section{Results}

Table 3 shows the basic statistics of the step error and postural sway variables for one minute and the results of the t-test. A significant difference was found between the step errors and all sway variables, being larger in the $40 \mathrm{bpm}$ tempo than in the $120 \mathrm{bpm}$ tempo. The ES observed was large values over 0.7 .

Table 3. Basic Statistics of the Step Error and Postural Sway Variables for One Minute and the Results of the T-Test

\begin{tabular}{lllllll}
\hline & \multicolumn{2}{c}{ 40bpm } & \multicolumn{3}{c}{ 120bpm } & \\
& Mean & SD & Mean & SD & t-value & ES \\
\hline step error $(\mathrm{sec})$ & 0.22 & 0.12 & 0.09 & 0.05 & $9.539 *$ & 1.38 \\
X-axis path length $(\mathrm{mm})$ & 147.5 & 17.9 & 119.4 & 12.0 & $17.094 *$ & 1.84 \\
Y-axis path length $(\mathrm{mm})$ & 98.3 & 24.0 & 79.8 & 28.8 & $6.301 *$ & 0.70 \\
total path length $(\mathrm{mm})$ & 196.6 & 27.0 & 157.1 & 21.5 & $13.003 *$ & 1.62 \\
peripheral area $\left(\mathrm{mm}^{2}\right)$ & 744.2 & 170.3 & 311.8 & 76.9 & $24.932 *$ & 3.27 \\
rectangular area $\left(\mathrm{mm}^{2}\right)$ & 990.0 & 233.1 & 410.5 & 102.7 & $23.584 *$ & 3.22 \\
\hline
\end{tabular}
$\mathrm{P}<0.05$

Table 4 shows the basic statistics of the step error and sway variables by the tempos and interval factors and the results of the two-way ANOVA (tempo $\mathrm{x}$ interval). The step error showed nonsignificant interaction, but the main effect for the tempo factor exhibited a significant one. Multiple comparison tests revealed that the means of three intervals were larger in the $40 \mathrm{bpm}$ tempo than in the $120 \mathrm{bpm}$ tempo (ES $=1.11-1.29$ ). Among the sway variables, a significant interaction was found only in peripheral area and rectangular area, and multiple comparison tests showed that the means of three intervals were larger in the $40 \mathrm{bpm}$ tempo than in the $120 \mathrm{bpm}$ tempo $(\mathrm{ES}=2.78-2.95)$. In addition, the means of both area variables were larger in the $0-20 \mathrm{sec}$ interval than in the 20-40 sec interval (ES = $0.37-0.38)$, and that of peripheral area was larger in the $0-20 \mathrm{sec}$ interval than in the 40-60 sec interval $(\mathrm{ES}=0.22)$. The X-axis path length showed a significant main effect in the tempo factor; in the multiple comparison tests, the means of three intervals were larger in the $40 \mathrm{bpm}$ tempo than in the $120 \mathrm{bpm}$ tempo $(\mathrm{ES}=1.76-1.80)$. Y-axis path length and total path length exhibited a significant main effect in the factors of tempo and interval. Multiple comparison tests also revealed that the means of three intervals were larger in the $40 \mathrm{bpm}$ tempo than in the $120 \mathrm{bpm}$ tempo $(\mathrm{ES}=0.61-1.59)$, and the mean of the $0-20 \mathrm{sec}$ interval was larger than that of the $40-60 \mathrm{sec}$ interval $(\mathrm{ES}=$ $0.16-0.23)$. Moreover, the mean of the Y-axis path length was larger in the 0-20 sec interval than in the 40-60 sec interval $(E S=0.15)$. 
Table 4. Basic Statistics of the Step Error and Sway Variables by the Tempos and Interval Factors and the Results of the Two-way ANOVA

\begin{tabular}{|c|c|c|c|c|c|c|c|c|}
\hline & & & \multicolumn{2}{|l|}{$40 \mathrm{bpm}$} & \multicolumn{2}{|c|}{$120 \mathrm{bpm}$} & \multirow[b]{2}{*}{ F-value } & \multirow[b]{2}{*}{ Post-hoc } \\
\hline & & & Mean & $\mathrm{SD}$ & Mean & $\mathrm{SD}$ & & \\
\hline \multirow[t]{3}{*}{ step error (sec) } & $0-20$ & $\mathrm{sec}$ & 0.22 & 0.14 & 0.10 & 0.07 & F1(tempo)95.019* & $0-20.20-40.40-60 \mathrm{sec}$ interval: $40 \mathrm{bpm}>$ \\
\hline & $\begin{array}{l}\text { interval } \\
20-40 \\
\text { interval }\end{array}$ & $\mathrm{sec}$ & 0.23 & 0.14 & 0.10 & 0.07 & F2(interval) $0.465^{*}$ & $120 \mathrm{bpm}$ \\
\hline & $\begin{array}{l}40-60 \\
\text { interval }\end{array}$ & $\mathrm{sec}$ & 0.24 & 0.14 & 0.10 & 0.06 & F3(interaction) $0.717^{*}$ & \\
\hline \multirow[t]{3}{*}{$\begin{array}{l}\mathrm{X} \text {-axis } \\
(\mathrm{mm})\end{array}$} & $\begin{array}{l}0-20 \\
\text { interval }\end{array}$ & $\mathrm{sec}$ & 49.3 & 6.4 & 39.8 & 3.9 & F1(tempo)292.203* & $\begin{array}{l}0-20.20-40.40-60 \mathrm{sec} \text { interval: } 40 \mathrm{bpm}> \\
120 \mathrm{bpm}\end{array}$ \\
\hline & $\begin{array}{l}20-40 \\
\text { interval }\end{array}$ & $\mathrm{sec}$ & 49.0 & 6.1 & 39.8 & 4.2 & F2(interval)0.318* & \\
\hline & $\begin{array}{l}40-60 \\
\text { interval }\end{array}$ & $\mathrm{sec}$ & 49.1 & 6.2 & 39.8 & 4.2 & $\mathrm{~F} 3$ (interaction) $0.224 *$ & \\
\hline \multirow[t]{3}{*}{$\begin{array}{l}\text { Y-axis path length } \\
(\mathrm{mm})\end{array}$} & $\begin{array}{l}0-20 \\
\text { interval }\end{array}$ & $\mathrm{sec}$ & 33.8 & 8.0 & 27.1 & 10.1 & F1(tempo)39.707* & $\begin{array}{l}0-20.20-40.40-60 \mathrm{sec} \text { interval: } 40 \mathrm{bpm}> \\
120 \mathrm{bpm}\end{array}$ \\
\hline & $\begin{array}{l}20-40 \\
\text { interval }\end{array}$ & $\mathrm{sec}$ & 31.9 & 8.5 & 26.4 & 9.4 & F2(interval)9.693* & $\begin{array}{l}\text { 40bpm: } 0-20 \mathrm{sec} \text { interval }>20-40.40-60 \mathrm{sec} \\
\text { interval }\end{array}$ \\
\hline & $\begin{array}{l}40-60 \\
\text { interval }\end{array}$ & $\mathrm{sec}$ & 32.6 & 8.5 & 26.2 & 9.1 & F3(interaction) $2.207^{*}$ & \\
\hline \multirow[t]{3}{*}{$\begin{array}{l}\text { total path length } \\
(\mathrm{mm})\end{array}$} & $\begin{array}{l}0-20 \\
\text { interval }\end{array}$ & $\mathrm{sec}$ & 66.3 & 9.5 & 52.8 & 7.5 & F1(tempo)169.077* & $\begin{array}{l}0-20.20-40.40-60 \mathrm{sec} \text { interval: } 40 \mathrm{bpm}> \\
120 \mathrm{bpm}\end{array}$ \\
\hline & $\begin{array}{l}20-40 \\
\text { interval }\end{array}$ & $\mathrm{sec}$ & 64.9 & 9.6 & 52.3 & 6.9 & F2(interval)4.897* & $\begin{array}{l}\text { 40bpm: } 0-20 \text { sec interval }>20-40 \mathrm{sec} \\
\text { interval }\end{array}$ \\
\hline & $\begin{array}{l}40-60 \\
\text { interval }\end{array}$ & $\mathrm{sec}$ & 65.3 & 9.2 & 52.0 & 7.4 & F3(interaction) $1.221^{*}$ & \\
\hline \multirow[t]{3}{*}{$\begin{array}{l}\text { peripheral } \\
\left(\mathrm{mm}^{2}\right)\end{array}$} & $\begin{array}{l}0-20 \\
\text { interval }\end{array}$ & $\mathrm{sec}$ & 261.2 & 73.4 & 104.7 & 26.3 & F1(tempo)621.617* & $\begin{array}{l}0-20.20-40.40-60 \mathrm{sec} \text { interval: } 40 \mathrm{bpm}> \\
120 \mathrm{bpm}\end{array}$ \\
\hline & $\begin{array}{l}20-40 \\
\text { interval }\end{array}$ & $\mathrm{sec}$ & 236.8 & 55.9 & 105.3 & 29.2 & F2(interval)5.409* & $\begin{array}{l}\text { 40bpm: } 0-20 \mathrm{sec} \text { interval }>20-40.40-60 \mathrm{sec} \\
\text { interval }\end{array}$ \\
\hline & $\begin{array}{l}40-60 \\
\text { interval }\end{array}$ & $\mathrm{sec}$ & 246.2 & 65.4 & 101.8 & 26.6 & F3(interaction)5.904* & \\
\hline \multirow[t]{3}{*}{$\begin{array}{l}\text { rectangular } \\
\left(\mathrm{mm}^{2}\right)\end{array}$} & $\begin{array}{l}0-20 \\
\text { interval }\end{array}$ & $\mathrm{sec}$ & 347.3 & 98.9 & 137.7 & 35.6 & F1(tempo)556.221* & $\begin{array}{l}0-20.20-40.40-60 \mathrm{sec} \text { interval: } 40 \mathrm{bpm}> \\
120 \mathrm{bpm}\end{array}$ \\
\hline & $\begin{array}{l}20-40 \\
\text { interval }\end{array}$ & $\mathrm{sec}$ & 313.3 & 79.7 & 138.7 & 39.3 & F2(interval) $4.568^{*}$ & $\begin{array}{l}\text { 40bpm: } 0-20 \text { sec interval }>20-40 \mathrm{sec} \\
\text { interval }\end{array}$ \\
\hline & $\begin{array}{l}40-60 \\
\text { interval }\end{array}$ & $\mathrm{sec}$ & 329.4 & 92.3 & 134.0 & 36.4 & F3(interaction) $5.690^{*}$ & \\
\hline
\end{tabular}

$\mathrm{P}<0.05$

Table 5-1 shows the correlation coefficient between the 40 and $120 \mathrm{bpm}$ tempos in each variable. The coefficient of the step error was significant but low $(\mathrm{r}=0.370)$; those of sway variables (X-and Y-axis path length, total path length, peripheral area, and rectangular area) were significant but of middle size ranging from 0.534 to 0.691 (shading in Table 4-1).

The $\mathrm{X}$-axis path length was insignificant with the Y-axis path length and both area variables, but other sway variables were mutually significant values $(r=0.371-0.592)$ of low-middle sizes. 
Table 5-1. Correlation Coefficient between the 40 and 120 bpm Tempos in Each Variable

\begin{tabular}{|c|c|c|c|c|c|c|}
\hline & (1) & (2) & (3) & (4) & (5) & (6) \\
\hline (1) step error & $0.370^{*}$ & & & & & \\
\hline (2) X-axis path length & 0.193 & $0.691^{*}$ & & & & \\
\hline (3) Y-axis path length & 0.049 & 0.026 & $0.627^{*}$ & & & \\
\hline (4) total path length & 0.125 & $0.371^{*}$ & $0.577^{*}$ & $0.534^{*}$ & & \\
\hline (5) peripheral area & 0.083 & 0.232 & $0.585^{*}$ & $0.460 *$ & $0.621 *$ & \\
\hline (6)rectangular area & 0.068 & 0.201 & $0.562 *$ & $0.425 *$ & $0.592 *$ & $0.574 *$ \\
\hline
\end{tabular}

Table 5-2 shows the correlation coefficients among variables in the 40 and $120 \mathrm{bpm}$ tempos. Most coefficients between the step error and sway variables were either insignificant or significant, but under 0.364. Those among sway variables in both tempos were significant and high $(\mathrm{r}=0.739-0.984)$, except between the X- and Y-axis path lengths. The former was a significant value $(\mathrm{r}=0.270)$ with the latter in the $40 \mathrm{bpm}$ tempo but insignificant in the 120 bpm tempo

Table 5-2. Correlation Coefficients among Variables in the 40 bpm Tempo (lower row) and 120 bpm Tempo (upper row)

\begin{tabular}{lllllll}
\hline & (1) & (2) & (3) & (4) & (5) & (6) \\
\hline (1) step error & & -0.176 & $0.293^{*}$ & 0.162 & 0.3648 & $0.355^{*}$ \\
(2) X-axis path length & $-0.270^{*}$ & & 0.089 & $0.563^{*}$ & $0.350^{*}$ & $0.332^{*}$ \\
(3) Y-axis path length & -0.036 & $0.434^{*}$ & & $0.867^{*}$ & $0.798^{*}$ & $0.793^{*}$ \\
(4) total path length & -0.169 & $0.852^{*}$ & $0.833^{*}$ & & $0.837^{*}$ & $0.821^{*}$ \\
(5) peripheral area & -0.195 & 0.713 & $0.759^{*}$ & $0.862^{*}$ & & $0.984^{*}$ \\
(6) rectangular area & -0.156 & 0.684 & $0.739^{*}$ & $0.830^{*}$ & $0.974^{*}$ & \\
\hline
\end{tabular}

\section{Discussion}

Leg strength and dynamic balance ability mainly relate to the step movement of the stipulated tempo step test. Hence, considering that people with superior ability can step stably regardless of a difference in tempo, the step error and the sway are small. Given that children that are underdeveloped in both abilities step with an unstable posture in the case of a slow tempo compared with a usual tempo due to the long supporting time by one leg, the step error the and postural sway become large and their relationship differs between the tempos.

In the results of this study, the step error showed that any mean of one minute and three intervals of $0-20 \mathrm{sec}, 20-40$ sec, and 40-60 sec (ES = 1.11-1.38) was larger in the $40 \mathrm{bpm}$ tempo than in the $120 \mathrm{bpm}$ tempo. All sway variables also obtained the same results as the step error $(\mathrm{ES}=0.61-3.27)$. Therefore, Hypotheses 1 and 2 were judged to have been adopted. According to Toyama et al. (1990), the $120 \mathrm{bpm}$ tempo is close to walking tempo. Bernstein (1967) reported that because stepping is a similar movement to walking, which is frequently experienced in daily life, it is a high movement of extremely learning level and automation level in adults. For a similar 120 bpm tempo to the walking tempo, even children can step stably, but for the $40 \mathrm{bpm}$ of the slow tempo, stepping while matching the tempo becomes difficult for them because the single-leg supporting time becomes long (approximately three times of the $120 \mathrm{bpm}$ tempo). Therefore, the step error and the postural sway become large.

In this study, we hypothesized that a slow tempo, step error, and postural sway are larger in the early interval than in the middle and/or the last intervals and that the measurement time for one minute is divided into three intervals. We also examined the differences among interval means. The results of multiple comparison tests revealed that the tempo error showed a nonsignificant difference among the interval mean in the tempos of 40 and $120 \mathrm{bpm}$, but the sway variables, except the $\mathrm{X}$-axis path length, exhibited a significant difference. In addition, only in the slow 40 bpm tempo, the mean of the early interval $(0-20 \mathrm{sec})$ was larger than that of the middle interval $(20-40 \mathrm{sec})$ or the last interval (40-60 sec). Wall and Charteris (1981) reported that practicing for more than one hour is required to get used to treadmill walking. In short, mastering unfamiliar movements that are different from daily ones takes time, and the early stage after the movement start may specifically affect the effect of familiar use. Considering that no significant difference was observed among the interval mean values in the $120 \mathrm{bpm}$ tempo, children had a difficult time stepping while matching the tempo during the early stage of measurement for the slow and unfamiliar $40 \mathrm{bpm}$ tempo; hence, postural sway was also affected. Hypothesis 3 was judged to have been adopted on sway variables, except the X-axis 
path length.

The relationship between the step errors in 40 and 120 bpm tempos was significant but low $(r=0.370)$. Shin and Demura (2009) examined the relationships between the step errors in the 40,60, and $120 \mathrm{bpm}$ tempos and the time of a $10 \mathrm{~m}$ line walking among the elderly. They reported that a significant and moderate correlation was found between 40 and $60 \mathrm{bpm}$ tempos, but not between $120 \mathrm{bpm}$ tempo and the other tempos. Step speeds of 40 and $120 \mathrm{bpm}$ tempos were quite different; thus, the abilities involved in both step movements were also different, and the relationship between their step errors was low.

The relationships between X-and Y-axis path lengths, total path length, peripheral area, and rectangular area of both tempos were low $(\mathrm{r}=0.534-0.691)$. The $\mathrm{X}$-axis path length was insignificant with variables, except the total path length. The mutual relationships of the other variables were also significant but not high $(\mathrm{r}=0.371-0.592)$. From these results, we inferred that even in the same stipulated tempo step test, children with inferior leg strength and dynamic balance have a more unstable posture in the $40 \mathrm{bpm}$ tempo than in the $120 \mathrm{bpm}$ tempo because the one-leg standing time becomes long. Hence, a difference in the tempo speed largely affects the step error and the postural sway. We also found that their relationships are not high. We thus confirmed Hypothesis 4.

Meanwhile, in the 40 and $120 \mathrm{bpm}$ tempos, the step errors showed little significant relationships with sway variables and even if they were significant, it was only under 0.364. Thus, regarding relationships among step errors and sway variables, we judged that there was no difference between both tempos. Hence, Hypothesis 5 was not supported. In addition to leg strength and dynamic balance ability, multiple factors, such as coordination ability and step timing, are involved in the step movements matching the tempo, and the related degree of these factors differs by individual. From the present results, given that the step error and the postural sway are influenced by many factors, regardless of the difference in tempo, their direct relationship is not that high.

Interrelationships among the sway variables in both tempos were high $(r=0.739-0.974)$, except those between both axis path lengths. The $\mathrm{X}$-axis path length showed a significant relationship $(\mathrm{r}=0.270)$ with the Y-axis path length in the $40 \mathrm{bpm}$ tempo but not in the $120 \mathrm{bpm}$ tempo. That is, their relationship differed between both tempos. Therefore, Hypothesis 5 was adopted only when the relationship between both axis path lengths was considered. Demura et al. (2001) reported significant and high relationships among postural sway variables regarding distance, area, and speed (above 0.90). Yasuda et al. (2012) reported high correlations among sway variables during stipulated tempo stepping $\left(r^{2}=0.88\right)$. Stepping with the $40 \mathrm{bpm}$ tempo has a long sway distance due to the long supporting time of a single leg, leading to a large sway area, whereas stepping with the $120 \mathrm{bpm}$ tempo has a short sway distance due to the short supporting time, leading to a small sway area. From the present results, the relationships among sway variables in both tempos are high, except $\mathrm{X}$-axis path length, and the total path length and area variables are more affected by the $\mathrm{Y}$-axis path length than by the $\mathrm{X}$-axis path length.

The stipulated tempo step tests employed in this study not only evaluates children's dynamic balance ability, but can also serve as basic material in the development of instructional methods for enhancing the same. In addition, the in-place step (stipulated tempo step) can easily be done in accordance with the teacher's hand-clapping gestures. Furthermore, whether the child is able to keep up with the tempo can be ascertained by the teacher's visual observation to a certain extent. Therefore, it may be possible to employ the in-place step as part of exercise and play activities in elementary school education.

The subjects in this study were children aged 6-11. Conducting the same research targeting youths with superior leg strength and dynamic balance ability or using tempos slower than $40 \mathrm{bpm}$ or faster than the $120 \mathrm{bpm}$ tempo will be necessary in the future. Examining the relationship between the stipulated tempo step tests and falling or stumbling in the elderly and developing a new test, which evaluates the fall avoidance ability of the elderly, may also be needed.

\section{Conclusion}

1. In the case of the stipulated tempo step test for children, the slow tempo (40 bpm) has a greater step error and postural sway than the fast tempo $(120 \mathrm{bpm})$; the sway during the early stage of the step is greater in the slow tempo than in the fast tempo.

2. The relationships between step errors and between the step error and sway variables of both tempos are low; hence, the ability related to the test using both tempos may be different.

3. Interrelationships among sway variables in both tempos are high, except those between $\mathrm{X}$ - and Y-axis path lengths. 
The relationship between both axis path lengths also differs in tempo.

\section{References}

Aoki, H., Demura, S., Kawabata, H., Sugiura, H., Uchida, Y., Xu, N., \& Murase, H. (2012). Evaluating the effects of open/closed eyes and age-related differences on center of foot pressure sway during stepping at a set tempo. AAR, 1, 72-77. https://doi.org/10.4236/aar.2012.13009

Apthorp, D., Nagle, F., \& Palmisano, S. (2014). Chaos in balance: non-linear measures of postural control predict individual variations in visual illusions of motion. PLoS One, 9, e113897. https://doi.org/10.1371/journal.pone.0113897

Bernstein, N. (1967). The Coordination and Regulation of Movements. Pergamon Press, Oxford.

Demura, S., Yamaji, S., Noda, M., Kitabayashi, T., \& Nagasawa, Y. (2001). Examination of Parameters Evaluating the Center of Foot Pressure in Static Standing Posture from the Viewpoints of Traial-to-trial Reliability and Interrelationships Among Parameters. Equilibrium research, 60, 44-55. https://doi.org/10.3757/jser.60.44

Demura, S., Kitabayashi, T., \& Aoki, H. (2008). Body-sway characteristics during a static upright posture in the elderly. Geriatr Gerontol Int, 8, 188-197. https://doi.org/10.1111/j.1447-0594.2008.00469.x

Demura, T., Demura, S., Uchiyama, M., Kitabayashi, T., \& Takahashi, K. (2015). Effect of shoes with rounded soft soles in the anterior-posterior direction on the center of pressure during static standing. Foot (Edinb), 25, 97-100. https://doi.org/10.1016/j.foot.2015.02.004

Hara, H., Yoshikawa, T., Nakao, Y., Wang, L., Suzuki, T., \& Fujimoto, S. (2007). The effects of aqua exercise on balance function in middle-aged woman. The Japanese Journal of Physical Fitness and Sports Medicine, 56, 357-364. https://doi.org/10.7600/jspfsm.56.357

Maribo, T., Stengaard-Pedersen, K., Jensen, L. D., Andersen, N. T., \& Schiøttz-Christensen, B. (2011). Postural balance in low back pain patients: Intra-session reliability of center of pressure on a portable force platform and of the one leg stand test. Gait Posture, 34, 213-217. https://doi.org/10.1016/j.gaitpost.2011.04.014

Shin, S., \& Demura, S. (2007). Effective tempo of the step test for dynamic balance ability in the elderly. $J$ Physiol Anthropol, 26, 563-567. https://doi.org/10.2114/jpa2.26.563

Shin, S., \& Demura, S. (2009). Relationship between the step test with stipulated tempos and gait ability in the elderly. J Physiol Anthropol, 28, 49-54. https://doi.org/10.2114/jpa2.28.49

Smith, A.W., Ulmer, F. F., \& Wong del, P. (2012). Gender differences in postural stability among children. J Hum Kinet, 33, 25-32. https://doi.org/10.2478/v10078-012-0041-5

Suzuki, Y., Nakata, Y., Kato, H., Tanabe, Y., Iwabuchi, S., \& Ishikawa, K. (2015). Association between age and dynamic balance capability assessed by use of force plates. The Japanese Journal of Physical Fitness and Sports Medicine, 64, 419-425. https://doi.org/10.7600/jspfsm.64.419

Toyama, H., \& Fujiwara, K. (1990). Interference of upper limbs exercise with different automatized levels. Physical Fitness Sports Med, 39, 44-52. https://doi.org/10.7600/jspfsm1949.39.44

Uchida, M., \& Nagura, T. (2014). The Relationship of Step Exercise with Single-leg Standing from the Perspective of Center of Gravity Sway. Rigakuryoho Kagaku, 29, 549-553. https://doi.org/10.1589/rika.29.549

Wall, J. C., \& Charteris, J. (1981). A kinematic study of long-term habituation to treadmill walking. Ergonomics, 24, 531-542. https://doi.org/10.1080/00140138108924874

Yasuda, T., Etoh, N., Araki, Y., Yamada, Y., Ide, R., Ohkawara, T., \& Kunihiro, T. (2012). A Dynamic Equilibrium Examination on Stabilometry (Foulage test) -Physiological Character of Normal Subjects-. Equilibrium Res, 71, 61-70. https://doi.org/10.3757/jser.71.61

Walters-Stewart, C., Rochefort, C., Longtin, A., Zemek, R., \& Sveistrup, H. (2018). Centre of pressure during quiet stance and dual-task one month after mild traumatic brain injury: In adolescents. Journal of Concussion, 2, 1-9. https://doi.org/10.1177/2059700218804917 


\section{Copyrights}

Copyright for this article is retained by the author(s), with first publication rights granted to the journal.

This is an open-access article distributed under the terms and conditions of the Creative Commons Attribution license (http://creativecommons.org/licenses/by/4.0/). 